\title{
Human leucocyte antigen class I in hormone receptor-positive, HER2-negative breast cancer: association with response and survival after neoadjuvant chemotherapy
}

\author{
Bruno Valentin Sinn ${ }^{1,2^{*}}$ D, Karsten E. Weber ${ }^{3}$, Wolfgang Daniel Schmitt ${ }^{1}$, Peter A. Fasching ${ }^{4}$, \\ William Fraser Symmans ${ }^{5}$, Jens-Uwe Blohmer ${ }^{6}$, Thomas Karn ${ }^{7}$, Eliane Tabea Taube ${ }^{1}$, Frederick Klauschen ${ }^{1,8}$, \\ Frederik Marmé ${ }^{9}$, Christian Schem ${ }^{10,11}$, Elmar Stickeler ${ }^{12}$, Beyhan Ataseven ${ }^{13,14}$, Jens Huober ${ }^{15}$, \\ Gunter von Minckwitz ${ }^{3}$, Barbara Seliger ${ }^{16}$, Carsten Denkert ${ }^{1,8,17 \dagger}$ and Sibylle Loibl ${ }^{3+}$
}

\begin{abstract}
Background: Clinical application of cancer immunotherapy requires a better understanding of tumor immunogenicity and the tumor microenvironment. HLA class I molecules present antigens to $\mathrm{CD}^{+}{ }^{+}$cytotoxic cells. Their loss or downregulation is frequently found in tumors resulting in reduced $T$ cell responses and worse prognosis.

Methods: We evaluated HLA class I heavy chain expression by immunohistochemistry in 863 biopsies (GeparTrio trial). Patients received neoadjuvant chemotherapy and adjuvant endocrine treatment if tumors were hormone receptor-positive $(\mathrm{HR}+)$. In parallel, the expression of HLA-A was analyzed using a microarray cohort of 320 breast cancer patients from the MD Anderson Cancer Center. We evaluated its association with clinical outcome, tumorinfiltrating lymphocytes (TILs), and immune cell metagenes.

Results: In HR+/HER2 - breast cancer, HLA class I heavy chain expression was associated with increased TILs and better response to chemotherapy ( $7 \%$ vs. $14 \% \mathrm{pCR}$ rate, $P=0.029$ ), but worse disease-free survival (hazard ratio (HR) $1.6(1.1-2.4) ; P=0.024)$. The effect was significant in a multivariate model adjusted for clinical and pathological variables (HR $1.7(1.1-2.6) ; P=0.016)$ and was confirmed by analysis of HLA-A in a microarray cohort. HLA-A was correlated to most immune cell metagenes. There was no association with response or survival in triple-negative or HER2+ disease.

Conclusions: The study confirms the negative prognostic role of lymphocytes in HR+ breast cancer and points at a complex interaction between chemotherapy, endocrine treatment, and tumor immunogenicity. The results point at a subtype-specific and potentially treatment-specific role of tumor-immunological processes in breast cancer with different implications in triple-negative and hormone receptor-positive disease.
\end{abstract}

Keywords: Breast cancer, HLA, Tumor-infiltrating lymphocytes

\footnotetext{
* Correspondence: bruno.sinn@charite.de

${ }^{\dagger}$ Carsten Denkert and Sibylle Loibl shared senior authorship.

${ }^{1}$ Department of Pathology, Charité - Universitätsmedizin Berlin, corporate

member of Freie Universität Berlin, Humboldt - Universität zu Berlin, and

Berlin Institute of Health, Berlin, Germany

${ }^{2}$ Berlin Institute of Health (BIH), Berlin, Germany

Full list of author information is available at the end of the article
}

(c) The Author(s). 2019 Open Access This article is distributed under the terms of the Creative Commons Attribution 4.0 International License (http://creativecommons.org/licenses/by/4.0/), which permits unrestricted use, distribution, and reproduction in any medium, provided you give appropriate credit to the original author(s) and the source, provide a link to the Creative Commons license, and indicate if changes were made. The Creative Commons Public Domain Dedication waiver (http://creativecommons.org/publicdomain/zero/1.0/) applies to the data made available in this article, unless otherwise stated. 


\section{Background}

Interactions between cancer cells and the host immune system are important for development, evolution, and progression of cancer [1]. They influence response to therapy and survival of patients and modulating these effects offer new approaches for cancer therapy.

Immune checkpoint inhibitor (CPI) therapy can enhance therapy response in advanced triple-negative breast cancer (TNBC) [2], and several clinical trials are ongoing. For a successful implementation of such strategies in clinical practice, a better understanding of tumor-immunological effects is necessary.

Evidence suggests a role of the immune system in breast cancer, as the quantity of tumor-infiltrating lymphocytes (TILs) is associated with better response to neoadjuvant chemotherapy and better patient outcome $[3,4]$. In a recent meta-analysis, TILs were associated with a higher probability of pathologic complete response (pCR) [5]. Abundant TILs were associated with a longer disease-free survival (DFS) in HER2+ breast cancer and TNBC. In contrast, TILs were associated with a shorter OS in patients with hormone receptor-positive, HER2-negative (HR+/HER2-) disease, pointing at differences according to breast cancer subtype.

Human leucocyte antigen (HLA) class I molecules are expressed on the surface of all nucleated cells and are encoded by the human leukocyte antigens HLA-A, HLA-B, and HLA-C. Their function is to present antigens to CD8+ cytotoxic $\mathrm{T}$ lymphocytes to recognize and eliminate infected or tumor cells [6]. Downregulation or loss of HLA class I expression is a frequent event in pathogen-infected and tumor cells as an effective mechanism to evade immune recognition [7, 8]. Deficient HLA class I expression can be mediated by promoter methylation [9], mutations in the HLA class I heavy chains $(\mathrm{HC}), \mathrm{b}_{2}$-microglobulin $\left(\mathrm{b}_{2}-\mathrm{m}\right)$ or APM components, loss of heterozygosity of HLA gene loci, and transcriptional regulation $[10,11]$. Molecular data suggest an impaired expression of components of the antigen presentation machinery (APM) as a mechanism of resistance to $\mathrm{T}$ cell response [12] and HLA class I abnormalities have been identified in tumors resistant to CPIs or adoptive $\mathrm{T}$ cell therapy [13].

Aim of this study was to evaluate the potential of HLA class I HC expression for prediction of response to neoadjuvant chemotherapy. To this end, we evaluated expression of HLA class I HC in a large cohort of breast cancer patients treated with anthracycline/ taxane-based neoadjuvant chemotherapy (within the GeparTrio trial $[14,15])$. We used a clinically annotated microarray cohort from the MD Anderson Cancer Center to validate the findings and investigate correlations with predefined metagenes [16] of different immune cell populations.

\section{Methods}

\section{GeparTrio trial cohort}

The neoadjuvant GeparTrio pilot [17] (NCT00544765) and main (NCT00544765) trials [14, 15] were prospective, randomized phase II and III trials including 2357 patients with breast cancer (cT2-4 cN0-3 cM0) recruited between 2001 and 2005. Patients received two cycles of docetaxel, adriamycin, and cyclophosphamide (TAC) and response was evaluated by ultrasound. Responders received four more cycles of TAC (pilot study) or were randomly assigned to four or six cycles of TAC (main study). Non-responders were randomized to receive either four cycles of TAC or four cycles of vinorelbine and capecitabine. HR+ was defined as $\geq 10 \%$ of tumor cells with estrogen receptor and/or progesterone receptor expression, as defined in the study protocol. HER2 positivity was determined by immunohistochemistry (HER2 score $3+$ ) and in situ hybridization where appropriate (HER2/CEP17 ratio > 2.2). Endocrine treatment for 5 years was planned for patients with $\mathrm{HR}+$ disease but was not part of the protocol. HER2 therapy was not available at that time. We used all samples with available material in the central biobank. Additional file 3: Table S1 lists the pathological and clinical characteristics of the patients. The extend of tumor-infiltrating lymphocytes (TILs) was estimated on H\&E-stained whole tissue slides as the area of tumor cells (for intratumoral TILs) or the stromal area (for stromal TILs) that is covered by lymphocytes [3].

\section{Immunohistochemistry of HLA class I antigens}

Immunohistochemical staining was performed on tissue microarrays using the anti-HLA class I antibody EMR-8-5 (dilution 1:600) recognizing the HLA class I HC HLA-A, HLA-B, and HLA-C (MBL, Woburn, MA, USA). The percentage of cells with membranous staining relative to all cancer cells was assigned to five categories: (0) $0 \%$, (1) 1$9 \%$, (2) $10-50 \%$, (3) $51-80 \%$, and (4) $81-100 \%$. The intensity of staining was assigned into four categories: (0) negative, (1) weak, (2) moderate, (3) strong. The groups for percentage and staining intensity were multiplied resulting in the immunoreactive score (IRS) ranging from 0 to 12. Based on the distribution of staining levels and patient outcome, a cut point (IRS > 2) was selected to optimize separation of curves in Kaplan-Meier analysis. In addition, the percentage of positively stained tumor cells was used continuously to evaluate the effect of HLA immunohistochemistry on clinical endpoints without a defined cut-off.

MD Anderson cancer center (MDACC) microarray cohort Gene expression data and clinical information of the MD Anderson Cancer Center cohort [18] (Affymetrix U133A microarrays) was downloaded from the GEO repository (GSE25066). A total of 320 tumors were $\mathrm{HR}+$ / 
HER2 - and patients received neoadjuvant chemotherapy and adjuvant endocrine treatment if $\mathrm{HR}+\mathrm{HR}$ positivity was defined as any number of stained cells. We applied an additional filter based on gene expression of ESR1 (probeset 205225_at) based on its bimodal distribution (> 10.45). In total, 267 patients met these criteria. Additional file 3: Table S2 lists the patient characteristics. HLA-A, HLA-B, and HLA-C metagenes were calculated as mean expression of the respective probesets (HLA-A: 215313_x_at, 213932_x_at; HLA-B: 211911_x_at, 209140_x_at, 208729_ x_at, HLA-C: 208812_x_at, 216526_x_at, 214459_x_at, 211799_x_at). Unsupervised cut-offs were chosen by assigning the same fraction of cases to the groups with high $(60 \%)$ and low $(40 \%)$ expression as in the GeparTrio dataset (HLA-A > 14.24, HLA-B > 13.63, and HLA-C > 13.69). Probesets 205225_at (estrogen receptor 1) and 208079_s_at (Aurora kinase A) were used to evaluate their association with HLA-A. Immune cell metagenes were calculated as the mean expression of cell-type-specific genes [16].

\section{Statistical methods}

Pathologic complete response (pCR) was defined as the absence of invasive cancer in breast and lymph nodes (ypT0/ypTis ypN0). In the MD Anderson cohort, response to chemotherapy was recorded using the residual cancer burden index [19]. Disease-free survival (DFS) was defined as time from study entry to local or distant recurrence or death from any cause, distant recurrencefree survival (DRFS) as the interval between diagnosis and distant recurrence or death from any cause, and overall survival (OS) as the time from study entry to death from any cause. To evaluate associations between clinical and pathological variables with HLA I, $\chi^{2}$ tests were used. Kaplan-Meier estimators and log-rank tests were used for survival analyses ( $\mathrm{R}$ package survival). The
A

HLA I Immunohistochemistry (GeparTrio)

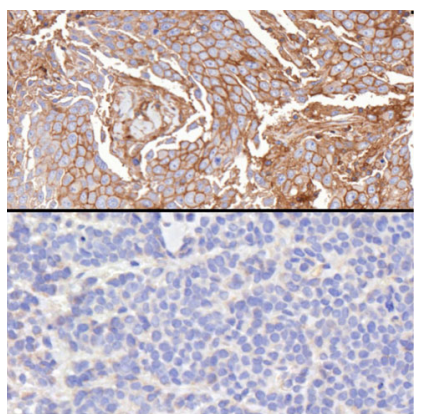

C

HLA I and tumor subtype (GeparTrio)

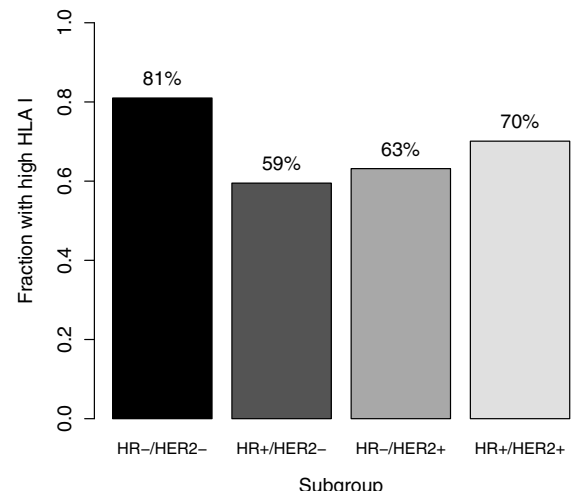

B

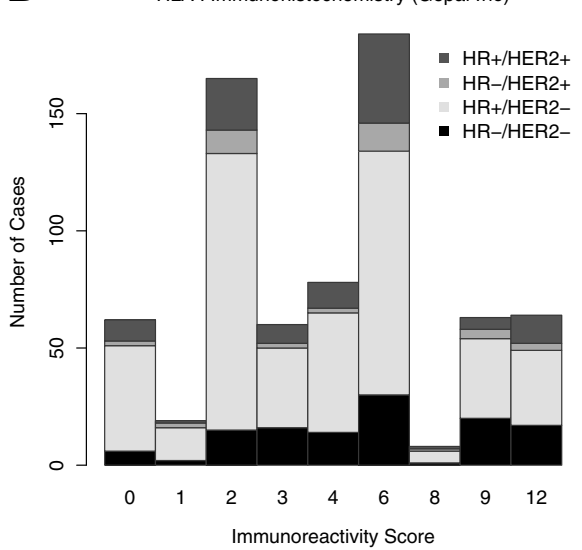

DLA I and patient characteristics (GeparTrio)

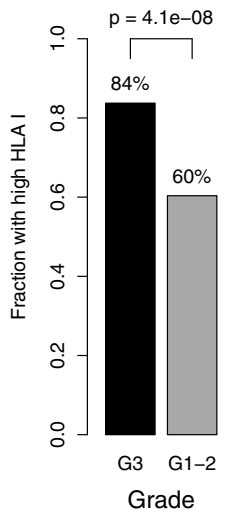

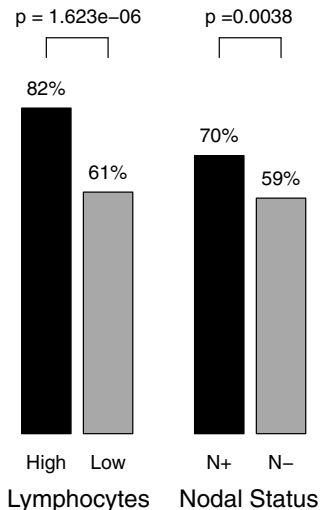

Fig. 1 HLA class I HC immunohistochemistry in the GeparTrio cohort: HLA class I HC immunohistochemistry showed a membranous staining pattern. Examples of a positive case and a negative case are shown in the upper and lower half, respectively (a). The data distribution of the immunoreactive score (IRS) as a function of staining quantity (percentage) and quality (intensity) is shown. The colored bars represent the different breast cancer subtypes (b). HLA I was more frequently high in HR-/HER2- breast cancer as compared to other subtypes (c). It was also associated with higher tumor grade, tumor-infiltrating lymphocytes, and nodal status (d) 
independent prognostic value was assessed in multivariate Cox regression analyses and likelihood ratio tests. Statistical computations were performed in $\mathrm{R}$ version 3.3.1 (R Development Core Team, Vienna, Austria). Significance was based on $P<0.05$ and 95\% CI estimates.

\section{Results}

\section{HLA class I expression in breast cancer}

A total of 732 cases from the GeparTrio trial were analyzed for HLA class I HC expression. In total, 669 cases (91\%) showed a positive staining; 63 were negative $(9 \%$, Fig. 1). We defined a cut-off to dichotomize HLA class I expression based on data distribution and patient outcome. This resulted in 480 cases with high expression (66\%) and 252 (34\%) with low expression across breast cancer subtypes. Additional file 3: Table S1 lists the patient and tumor characteristics of the GeparTrio study cohort that was evaluated for HLA class I HC expression (and Additional file 3: Table S2 the characteristics of the HR+/HER2 - cohort).

\section{Association with clinical and pathological tumor characteristics}

The frequency of HLA class I HC expression was higher in TNBC compared to other subtypes ( $81 \%$ in TNBC, Fig. 1$)$, in patients with node-positive disease $(P=0.004)$ and in tumors with a higher histological grade $(P<0.001)$. High levels of HLA class I HC expression was associated with extensive stromal TILs in the entire cohort $(P<0.001$, Fig. 1$)$ and within HR+/HER2- tumors $(P<0.001)$, where both the extent of intratumoral and stromal TILs were associated with high HLA class I HC expression (Fig. 2). There was no significant association with HER2 status, clinical T stage (T1-2 vs. T34), and histological subtype (no special type vs. lobular; data not shown).

\section{Association of HLA class I HC expression with clinical endpoints}

High HLA class I HC expression was associated with a higher rate of pathologic complete response $(\mathrm{pCR})$ in patients with HR+/HER2- disease ( $7 \%$ vs. $14 \%, P=0.029$,
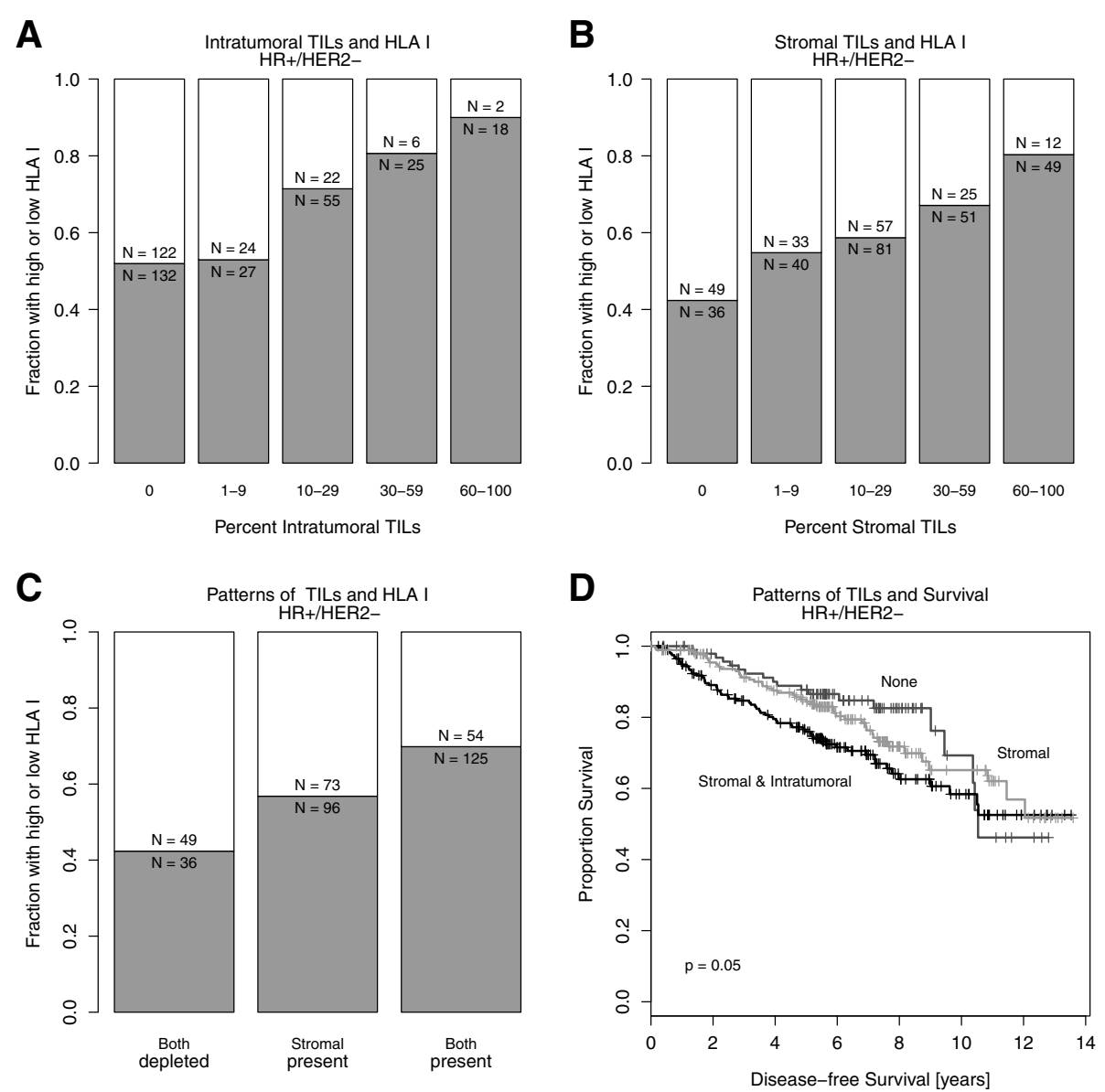

Fig. 2 HLA class I HC expression in HR+/HER2- breast cancer and patterns of tumor-infiltrating lymphocytes (TILs): the fraction of tumors with high (gray) and low (white) HLA class I HC expression are shown according to the quantity of intratumoral TILs (a), stromal TILs (b) and the combination of the two patterns (c). TILs and their distribution and their association with progression-free survival in HR+/HER2- breast cancer (d) 
Table 1), even after adjustment for clinical and pathological characteristics, but not in other subtypes (Table 1). High HLA class I expression was associated with shorter DFS in HR+/HER2- breast cancer $(P=$ 0.024 , Fig. 3, Table 1), but not in other subtypes (Table 1). In patients with HR+/HER2- breast cancer, HLA class I HC expression was prognostic when stratified for nodal status (Fig. 3) and in a multivariate analysis adjusted for clinical and pathological characteristics (Table 1). In an exploratory analysis within HR+/HER2breast cancer, HLA class I HC was associated with higher risk of relapse in patients with grade 1-2 tumors, patients with residual disease after neoadjuvant chemotherapy, in patients with non-lobular breast cancer and those without extensive intratumoral lymphocytes (Additional file 1: Figure S1). We also used the percentage of cells positively stained for HLA I to evaluate its association with clinical endpoints without a predefined cut-off (Additional file 3: Table S3).

\section{HLA I expression in an independent dataset}

For validation, we used data from a clinically annotated, publicly available dataset of 267 hormone HR+/HER2breast cancer patients with chemo-endocrine treatment [18] that shares characteristics with the GeparTrio cohort (Additional file 3: Table S2). We evaluated the expression of HLA-A, HLA-B, and HLA-C using the continuous data and defined cut points corresponding to the observed frequencies of high vs. low HLA class I expression in the IHC dataset (Table 2, Fig. 3). HLA-A, HLA-B, and HLA-C were strongly correlated (Additional file 2: Figure S2). HLA-A, but not HLA-B or HLA-C, was associated with longer distant recurrence-free survival but not with response to therapy (Table 2, Fig. 3).

\section{Association of HLA class I expression with immune cell populations}

The association of HLA-A expression with previously described immune-cell-specific metagenes [16] was evaluated in the microarray-based dataset to better understand the association with the immunological tumor infiltrate (Additional file 3: Table S4). Within the HR+/HER- subset, positive correlations of the HLA metagene were found with all signatures (Additional file 3: Table S4). The strongest associations were detected for the $\mathrm{T}$ cell signatures $\left(\rho_{\mathrm{P}}=0.553\right)$, myeloid dendritic cells $\left(\rho_{\mathrm{P}}=0.488\right)$, and cytotoxic lymphocyte signatures $\left(\rho_{\mathrm{P}}=0.471\right)$.

\section{Immune cell populations and patient outcome}

Univariate Cox and logistic regression were performed to evaluate the predictive and prognostic influence of the different immune cell populations in HR+/HER2breast cancer. The metagene representing neutrophils was associated with reduced response to neoadjuvant
Table 1 Univariate Cox and logistic regression within tumor subtypes and multivariate Cox and logistic regression in patients with HR+/HER2- breast cancer for comparing tumors with high vs. low HLA class I immunohistochemistry

\begin{tabular}{lllll}
\hline \multicolumn{3}{l}{ Univariate Cox regression-disease-free survival (GeparTrio) } & \\
Subtype & $\mathrm{MHCl}$ & $\mathrm{HR}$ & $95 \% \mathrm{Cl}$ & $P$ \\
HR+/HER2- & High vs. low & 1.590 & $1.062-$ & 0.024 \\
& & & 2.380 & \\
HR-/HER2- & High vs. low & 0.649 & $0.315-$ & 0.241 \\
& & & 1.336 & \\
HR+/HER2+ & High vs. low & 2.486 & $0.956-$ & 0.062 \\
& & & 6.467 & \\
HR-/HER2+ & High vs. low & 1.015 & $0.304-$ & 0.981 \\
& & & 3.384 &
\end{tabular}

Multivariate Cox regression—disease-free survival (GeparTrio; HR+/HER2-)

$\begin{array}{lllll} & & \text { HR } & 95 \% \mathrm{Cl} & P \\ \text { Response } & \text { pCR vs. RD } & 0.457 & 0.225- & 0.029 \\ & & & 0.925 & \\ \text { CT stage } & \text { CT3-4 vs. CT1-2 } & 2.270 & 1.522- & < \\ & & & 3.385 & 0.001 \\ \text { CN stage } & \text { CN+ vs. CN- } & 2.026 & 1.335- & 0.001 \\ & & & 3.075 & \\ \text { Therapy } & \text { Resp. guided vs. } & 0.945 & 0.638- & 0.777 \\ & \text { standard } & & 1.400 & \\ \text { Grade } & \text { G3 vs. G1-2 } & 1.733 & 1.024- & 0.041 \\ & & & 2.932 & \\ \text { Age } & \text { Age } \geq 50 \text { vs. }<50 & 1.252 & 0.838- & 0.272 \\ & & & 1.870 & \\ \text { HLA class I } & \text { High vs. low } & 1.701 & 1.105- & 0.016 \\ \text { HC } & & & 2.618 & \end{array}$

Univariate logistic regression-pCR (GeparTrio)

$\begin{array}{lllll}\text { Subtype } & \text { MHCl } & \text { HR } & 95 \% \mathrm{Cl} & P \\ \text { HR+/HER2- } & \text { High vs. low } & 2.226 & 1.154- & 0.022 \\ & & & 4.585 & \\ \text { HR-/HER2- } & \text { High vs. low } & 0.852 & 0.332- & 0.744 \\ & & & 2.316 & \\ \text { HR+/HER2+ } & \text { High vs. low } & 2.022 & 0.728- & 0.202 \\ & & & 6.590 & \\ \text { HR-/HER2+ } & \text { High vs. low } & 0.857 & 0.220- & 0.823 \\ & & & 3.385 & \end{array}$

Multivariate logistic regression-pCR (GeparTrio; HR+/HER2-)

\begin{tabular}{|c|c|c|c|c|}
\hline & & $H R$ & $95 \% \mathrm{Cl}$ & $P$ \\
\hline CT stage & cT3-4 vs. cT1-2 & 0.946 & $\begin{array}{l}0.447- \\
1.903\end{array}$ & 0.880 \\
\hline cN stage & $\mathrm{cN}+$ vs. $\mathrm{cN}-$ & 1.107 & $\begin{array}{l}0.579- \\
2.135\end{array}$ & 0.760 \\
\hline Therapy & $\begin{array}{l}\text { Resp. guided vs. } \\
\text { standard }\end{array}$ & 0.842 & $\begin{array}{l}0.444- \\
1.578\end{array}$ & 0.593 \\
\hline Grade & G3 vs. G1-2 & 2.804 & $\begin{array}{l}1.348- \\
5.644\end{array}$ & 0.005 \\
\hline Age & Age $\geq 50$ vs. $<50$ & 0.564 & $\begin{array}{l}0.297- \\
1.055\end{array}$ & 0.075 \\
\hline $\begin{array}{l}\text { HLA class I } \\
\text { HC }\end{array}$ & High vs. low & 2.132 & $\begin{array}{l}1.057- \\
4.603\end{array}$ & 0.042 \\
\hline
\end{tabular}



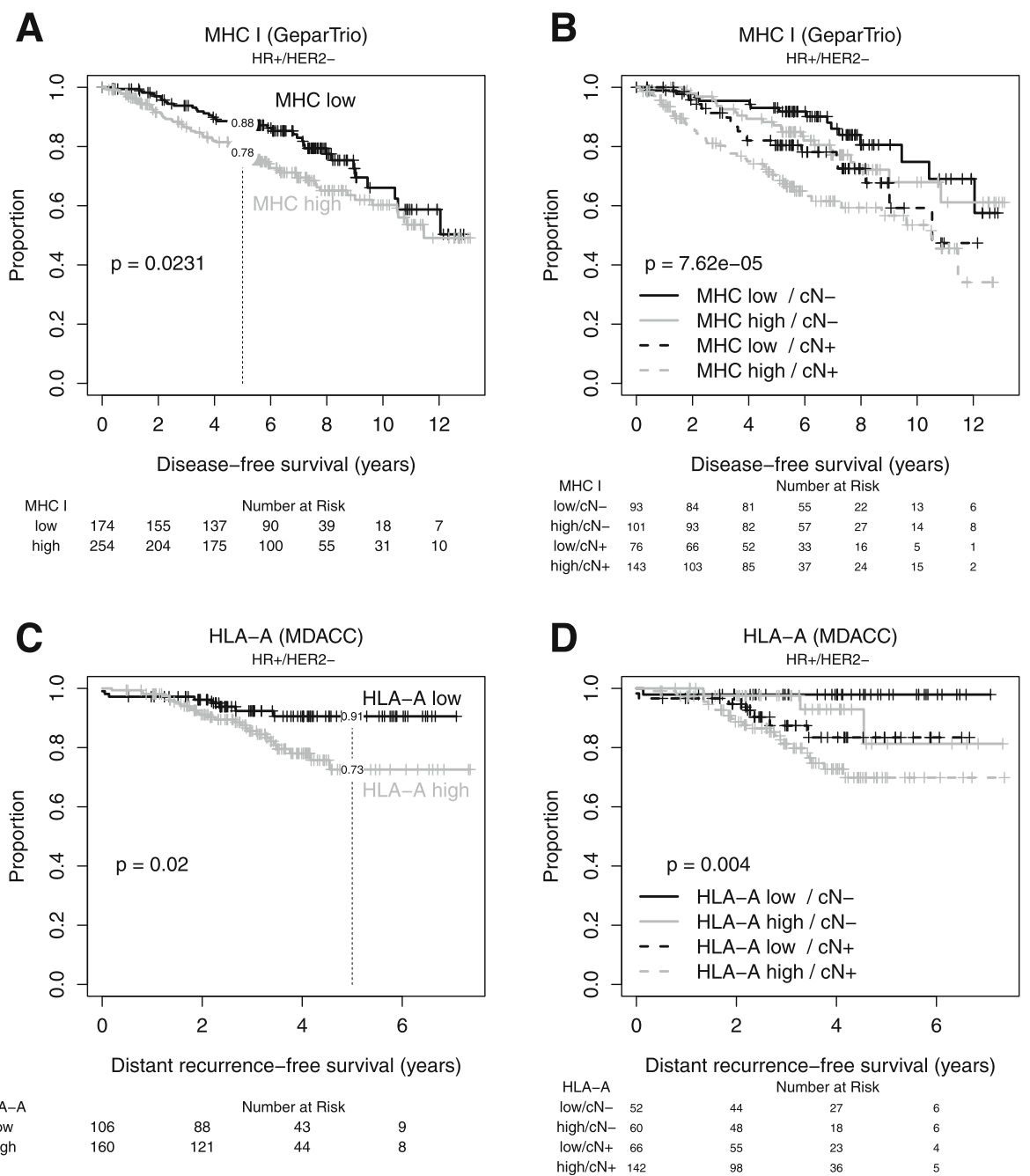

Fig. 3 HLA class I HC immunohistochemistry in GeparTrio and HLA-A mRNA analysis in the MDACC cohort: in GeparTrio, HLA class I immunohistochemistry was associated with shorter disease-free survival $(\mathbf{a}, \mathbf{b})$ in HR+/HER2- tumors. For the HR+/HER2- microarray data, we chose a cut-off for HLA-A without prior knowledge of patients' outcome to assign the same fraction of patients to each group as in the immunohistochemistry dataset. The effect on survival could be confirmed $(\mathbf{c}, \mathbf{d})$

chemotherapy, while the metagene for the monocytic lineage was associated with better response (Fig. 4). The HLA-A metagene, the monocytic lineage, and the B lineage were associated with higher risk of distant relapse.

\section{Association of HLA class I expression with ESR1 and Aurora kinase $\mathrm{A}$ expression}

To evaluate subtyping effects of HLA-A expression, we correlated the expression with estrogen receptor 1 (ESR1) and aurora kinase A (AUKRA) as a marker of proliferation [20]. There was no strong association of HLA-A with either gene $\left(\rho_{\mathrm{P}}=-0.164\right.$ and -0.122 , respectively; Additional file 3: Table S4).

\section{Discussion}

HLA class I HC expression as evaluated by IHC is predictive for better response and worse DFS and OS in HR+/HER2- breast cancer in a large clinical trial cohort. It was positively associated with TILs and independently prognostic for patient survival adjusted for other clinical and pathological factors. It was neither predictive nor prognostic in TNBC or HER2+ disease. We confirmed the prognostic value of HLA-A expression in an independent microarray-based dataset.

HLA class I HC expression was associated with clinical and pathological tumor characteristics indicative of a more aggressive tumor biology such as the presence of lymph node metastases and high histopathological grade. 
Table 2 Univariate Cox and logistic regression analyses to predict distant recurrence-free survival using the dichotomized and continuous expression data of HLA-A, HLA-B, and HLA-C, respectively. HLA-A, but not $H L A-B$ and $H L A-C$, is predictive for patient survival, but not for response to neoadjuvant treatment Univariate Cox regression-distant recurrence-free survival (MDACC)

\begin{tabular}{|c|c|c|c|c|}
\hline \multirow{2}{*}{ HLA-A } & \multirow[b]{2}{*}{ High vs. low } & \multirow{2}{*}{$\begin{array}{l}\mathrm{HR} \\
2.493\end{array}$} & \multirow{2}{*}{$\begin{array}{l}95 \% \mathrm{Cl} \\
1.131-5.494\end{array}$} & \multirow{2}{*}{$\begin{array}{l}P \\
0.023\end{array}$} \\
\hline & & & & \\
\hline HLA-B & High vs. low & 2.042 & $0.956-4.358$ & 0.065 \\
\hline HLA-C & High vs. low & 1.477 & $0.723-3.016$ & 0.284 \\
\hline \multicolumn{5}{|c|}{ Jnivariate logistic regression—pCR (MDACC) } \\
\hline & & $\mathrm{HR}$ & $95 \% \mathrm{Cl}$ & P \\
\hline HLA-A & High vs. low & 1.722 & $0.699-4.289$ & 0.234 \\
\hline HLA-B & High vs. low & 1.373 & $0.552-3.383$ & 0.487 \\
\hline HLA-C & High vs. low & 1.373 & $0.552-3.383$ & 0.487 \\
\hline
\end{tabular}

Univariate Cox regression-distant recurrence-free survival (MDACC)

$\begin{array}{lllll} & & \text { HR } & 95 \% \mathrm{Cl} & P \\ \text { HLA-A } & \text { Continuous } & 1.576 & 1.079-2.303 & 0.019 \\ \text { HLA-B } & \text { Continuous } & 1.364 & 0.973-1.912 & 0.072 \\ \text { HLA-C } & \text { Continuous } & 1.359 & 0.941-1.965 & 0.102\end{array}$

Univariate logistic regression-pCR (MDACC)

\begin{tabular}{lllll} 
& & HR & $95 \% \mathrm{Cl}$ & $P$ \\
HLA-A & Continuous & 1.346 & $0.857-2.103$ & 0.192 \\
HLA-B & Continuous & 1.367 & $0.887-2.132$ & 0.160 \\
HLA-C & Continuous & 1.519 & $0.948-2.466$ & 0.085 \\
\hline
\end{tabular}

We considered that this might explain the better response, but worse DFS that was associated with high HLA class I HC expression. However, there was no strong association of HLA-A expression with either ESR1 expression or AURKA expression. HLA was prognostic in a multivariate analysis adjusted for clinical and pathological tumor characteristics including nodal status and tumor grade. Thus, the effects could not be explained by these correlations alone.

The main caveat of the study is that we cannot evaluate the role of HLA class I expression for sensitivity to different treatments, as patients received both cytotoxic and endocrine therapy. Also, we cannot provide data on HLA class I in its most relevant context of therapeutic immunomodulation.

Another major limitation of the study is the lack of an independent validation cohort, especially to validate the cut-point used for immunohistochemistry. Even though the GeparTrio and MD Anderson datasets share clinical and pathological features, a different technology is used to detect biomarkers on different biological levels. This approach comes with limitations, both on the technical and clinical levels. An important clinical difference is that in GeparTrio, hormone receptor positivity was defined as $\geq 10 \%$ of tumor cells stained for estrogen or progesterone receptor, in the MD Anderson cohort, as any stained tumor cells. As it is known that low hormone receptor-positive cases may resemble triple-negative disease on the molecular level and clinically [21, 22],

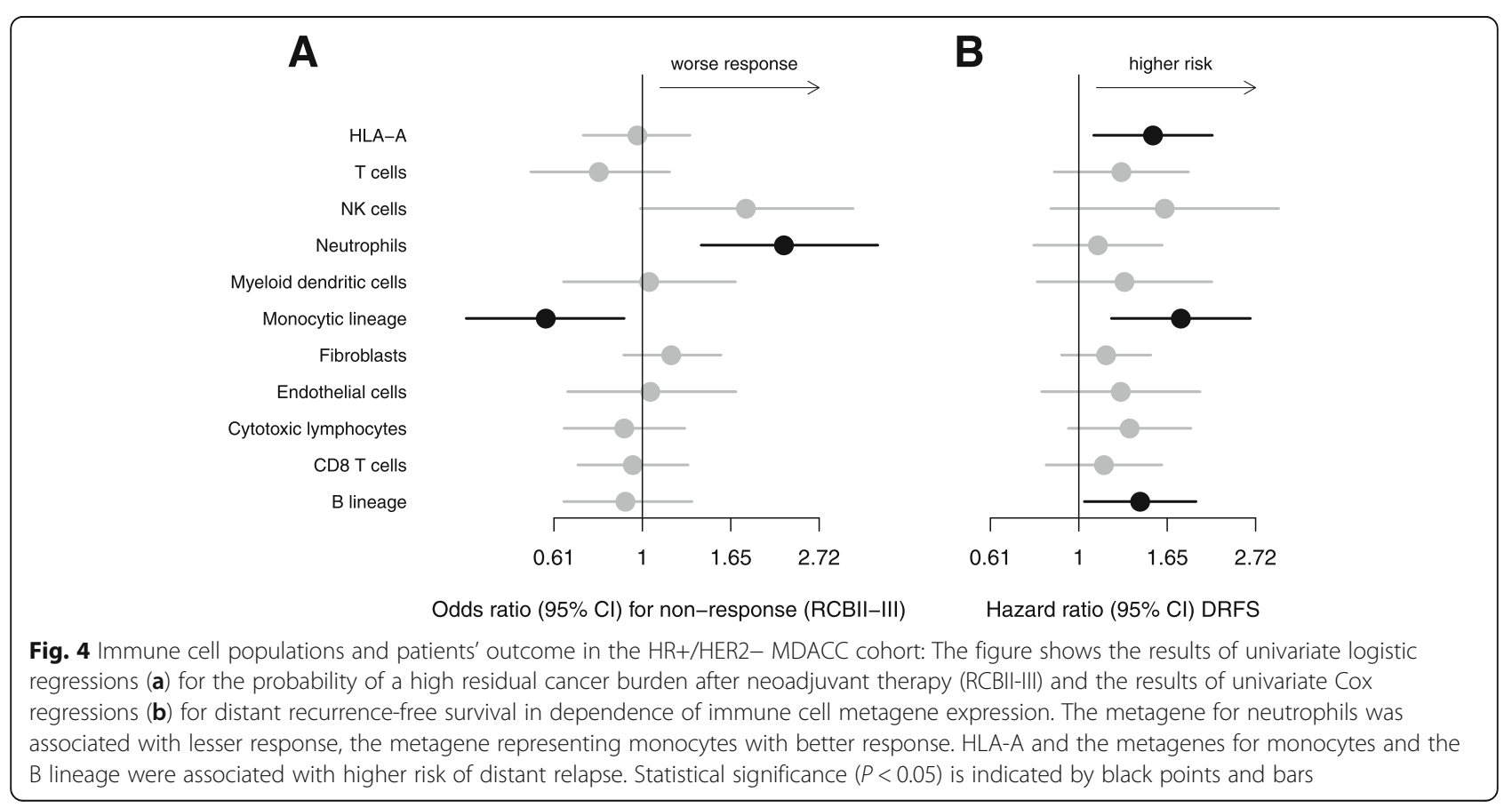


this might influence the comparability of the cohorts. We have therefore applied an additional filter to exclude cases with low ESR1 expression from the MD Anderson dataset.

With these limitations in mind, we believe it is a strength of our study that we are able to show the prognostic relevance of HLA in different cohorts and on the protein as well as mRNA level, which points out the role of HLA as a relevant parameter for the description of the tumor microenvironment.

HLA class I expression was previously evaluated by immunohistochemistry in 212 breast cancer samples not stratified for subtype [23]. The frequency of strong staining (32.5\%) was comparable to our data, but HLA class I was associated with a longer DFS across subtypes. In other cancers, like colorectal [24] and non-small cell lung cancer [25], HLA class I expression was positively associated with survival.

For patients with an excellent response to chemotherapy, we observed a trend towards better survival if HLA class I HC expression was high. However, this subgroup was very small, as most patients with $\mathrm{HR}+/$ HER2breast cancer do not show a complete response. In our recent meta-analysis of TILs in breast cancer [5], TILs were associated with better response to chemotherapy in HR+/HER2- disease, but shorter DFS and OS. The effect was strongest in large tumors and in patients who did not have an excellent response to chemotherapy. Our observations in this study are in line with our current findings, pointing at an interaction between lymphocytes and cytotoxic and endocrine treatment effects.

All of the analyzed immune cell metagenes were positively correlated with HLA-A expression. Only HLA-A, and the monocytic and $\mathrm{B}$ cell lineages were statistically significantly associated with higher risk, but as trends and correlations were similar for the different immune cell populations, we found it not possible to pinpoint HLA-A effects to one or more specific immune cell type based on these data.

Patient outcome in HR+/HER2- disease is a function of resistance and sensitivity to neoadjuvant cytotoxic therapy, adjuvant endocrine treatment, and natural biology. Because response to chemotherapy was better in patients with tumors with high HLA class I, we hypothesized that the shorter DFS might be due to an interaction with endocrine treatment. Tumors with a high TIL density might be less responsive to endocrine treatment by unknown mechanisms unrelated to ESR1 expression. Interestingly, immune gene modules can predict poor antiproliferative response to aromatase inhibitors [26, 27]. In a large study on patients receiving endocrine therapy alone, HLA class I was not prognostic, but the presence of FOXP3-positive cells was associated with better survival [28].

\section{Conclusions}

We demonstrate how HLA class I expression can be used to predict worse outcome in HR+/HER2- breast cancer. The results point at a subtype-specific and potentially treatment-specific role of tumor-immunological processes in breast cancer with different implications in triple-negative and hormone receptor-positive disease. Further studies are necessary to understand the underlying mechanisms as a foundation for the potential application of immunotherapy in HR+ breast cancer.

\section{Supplementary information}

Supplementary information accompanies this paper at https://doi.org/10. 1186/s13058-019-1231-z.

Additional file 1: Figure S1. HLA class I HC immunohistochemistry in
the GeparTrio cohort: In this exploratory subgroup analysis, univariate Cox
regression models were fit to evaluate the prognostic impact of the
dichotomized HLA class I data on progression-free survival (A) and overall
survival (B) in HR+/HER2- breast cancer. HLA class I was a negative prognostic
marker for disease-free survival across all patients, in those with a lower tumor
grade, in tumors without excellent response to chemotherapy, in patients
with no special type histopathology and in patients with tumors without
extensive lymphocytes. We observed a trend in the opposite direction for
patients with high-grade tumors, those with excellent response to
chemotherapy and lobular breast cancer. HLA class I was associated with
shorter overall survival in patients without excellent response (B).
Additional file 2: Figure S2. HLA-A, HLA-B and HLA-C expression in the
MDACC microarray cohort: A paired scatterplot illustrating the association
of the HLA metagenes. The upper right panels show Spearman's $\rho$.
Additional file 3: Table S1. Patient and tumor characteristics of the
German Breast Group GeparTrio cohort that was evaluated by
immunohistochemistry for HLA class I HC expression compared to
frequencies in the overall study population. Table S2. Patient and tumor
characteristics of the HR+/HER2- subset of the GeparTrio cohort
(immunohistochemistry) and the MD Anderson Cancer Center cohort
(Affymetrix U133A microarrays). Table S3. Univariate Cox and logistic
regression within tumor subtypes and multivariate Cox and logistic
regression in patients with HR+/HER2- breast cancer using the
percentage of tumor cells positively stained for MHC I. Table S4.
Correlations (Spearman's $\rho$ ) of the immune cell metagenes with HLA-A
expression, ESR1 and AURKA expression as a surrogate marker for
proliferation.

\section{Acknowledgements}

We would like to thank the patients who gave consent to participate in the GeparTrio trial and its translational research program.

\section{Authors' contributions}

BVS, CD, and SL conceived and planned the experiments. KEW, PAF, TK, FM, $C S, E S$, and GVM were involved in planning and approval of the study. JUB, FM, CS, ES, BA, and JH provided the materials. WFS provided the data. BVS, $C D$, WDS, ETT, and FK reviewed histological slides and evaluated the immunohistochemical stains. BVS analyzed the data. BVS and BS wrote the manuscript. All authors interpreted the results, edited, and approved the manuscript.

\section{Funding}

This work was supported by the German Cancer Aid within the TransLUMINAL-B grant; and the German Cancer Consortium (DKTK); and the BMBF within the TH4Response grant (JTC 2014-121). BVS is participant in the BIH Charité Clinician Scientist Program funded by the Charité - Universitätsmedizin Berlin and the Berlin Institute of Health.

Availability of data and materials Not applicable. 


\section{Ethics approval and consent to participate}

All patients gave informed consent for the use of tissue for research purposes and biomarker assessment. Ethical approval was obtained for all clinical centers and from the institutional review board of the Charité

\section{Consent for publication}

Not applicable.

\section{Competing interests}

The authors declare that they have no competing interests.

\section{Author details}

${ }^{1}$ Department of Pathology, Charité - Universitätsmedizin Berlin, corporate member of Freie Universität Berlin, Humboldt - Universität zu Berlin, and Berlin Institute of Health, Berlin, Germany. ${ }^{2}$ Berlin Institute of Health (BIH), Berlin, Germany. ${ }^{3}$ German Breast Group Forschungs GmbH, Neu-Isenburg, Germany. ${ }^{4}$ Department of Gynecology, University Hospital Erlangen, Erlangen, Germany. ${ }^{5}$ Department of Translational Molecular Pathology, The University of Texas - MD Anderson Cancer Center, Houston, TX, USA. ${ }^{6}$ Department of Gynecology with Breast Cancer, Charité Universitätsmedizin Berlin, corporate member of Freie Universität Berlin Humboldt - Universität zu Berlin, and Berlin Institute of Health, Berlin, Germany. ${ }^{7}$ Department of Gynecology and Obstetrics, University Hospital Frankfurt, Frankfurt, Germany. ${ }^{8}$ German Cancer Consortium (DKTK) Partner Site Berlin, Berlin, Germany. ${ }^{9}$ Department of Gynecology and Obstetrics, University Hospital Heidelberg, Heidelberg, Germany. ${ }^{10}$ Department of Gynecology and Obstetrics, University Hospital Schleswig-Holstein, Kiel, Germany. ${ }^{11}$ Mammazentrum Hamburg, Hamburg, Germany. ${ }^{12}$ Department of Gynecology and Obstetrics, RWTH Aachen, Aachen, Germany. ${ }^{13}$ Department of Gynecology and Gynecologic Oncology, Kliniken Essen Mitte, Essen, Germany. ${ }^{14}$ Department of Obstetrics and Gynecology, University Hospital, LMU Munich, Munich, Germany. ${ }^{15}$ Department of Gynecology and Breast Medical Oncology, Universitätsklinikum UIm, Ulm, Germany. ${ }^{16}$ Institute of Medical Immunology, Martin Luther University Halle-Wittenberg, Halle, Germany. ${ }^{17}$ Department of Pathology, University Hospital Marburg, Marburg, Germany.

Received: 29 April 2019 Accepted: 18 November 2019 Published online: 11 December 2019

\section{References}

1. Fridman WH, Zitvogel L, Sautès-Fridman C, Kroemer G. The immune contexture in cancer prognosis and treatment. Nat Rev Clin Oncol. 2017;14: 717-34

2. Nanda R, Chow LQM, Dees EC, Berger R, Gupta S, Geva R, et al. Pembrolizumab in patients with advanced triple-negative breast cancer: phase Ib keynote-012 study. J Clin Oncol. 2016;34:2460-7.

3. Denkert C, Loibl S, Noske A, Roller M, Müller BM, Komor M, et al. Tumorassociated lymphocytes as an independent predictor of response to neoadjuvant chemotherapy in breast cancer. J Clin Oncol. 2010;28:105-13.

4. Denkert C, von Minckwitz G, Brase JC, Sinn BV, Gade S, Kronenwett R, et al. Tumor-infiltrating lymphocytes and response to neoadjuvant chemotherapy with or without carboplatin in human epidermal growth factor receptor 2positive and triple-negative primary breast cancers. J Clin Oncol. 2015;33:983-91.

5. Denkert C, von Minckwitz G, Darb-Esfahani S, Lederer B, Heppner BI, Weber $\mathrm{KE}$, et al. Tumour-infiltrating lymphocytes and prognosis in different subtypes of breast cancer: a pooled analysis of 3771 patients treated with neoadjuvant therapy. Lancet Oncol. 2018;19:40-50.

6. Rock KL, Reits E, Neefjes J. Present yourself! By MHC class I and MHC class II molecules. Trends Immunol. 2016;37:724-37.

7. Vinay DS, Ryan EP, Pawelec G, Talib WH, Stagg J, Elkord E, et al. Immune evasion in cancer: mechanistic basis and therapeutic strategies. Semin Cancer Biol. 2015;35:S185-98.

8. Cai L, Michelakos T, Yamada T, Fan S, Wang X, Schwab JH, et al. Defective HLA class I antigen processing machinery in cancer. Cancer Immunol Immunother. 2018;67:999-1009.

9. Luo N, Nixon MJ, Gonzalez-Ericsson PI, Sanchez V, Opalenik SR, Li H, et al. DNA methyltransferase inhibition upregulates MHC-I to potentiate cytotoxic T lymphocyte responses in breast cancer. Nat Commun. 2018;9:248.
10. Garrido F, Aptsiauri N, Doorduijn EM, Garcia Lora AM, van Hall T. The urgent need to recover $\mathrm{MHC}$ class I in cancers for effective immunotherapy. Curr Opin Immunol. 2016;39:44-51.

11. Seliger B. Novel insights into the molecular mechanisms of HLA class I abnormalities. Cancer Immunol Immunother. 2012;61:249-54.

12. Rooney MS, Shukla SA, Wu CJ, Getz G, Hacohen N. Molecular and genetic properties of tumors associated with local immune cytolytic activity. Cell. 2015;160:48-61.

13. Nowicki TS, Hu-lieskovan S, Ribas A. Mechanisms of resistance to PD-1 and PD-L1 blockade. Cancer. 2018;24:47-53

14. Von Minckwitz G, Kümmel S, Vogel P, Hanusch C, Eidtmann H, Hilfrich J, et al. Intensified neoadjuvant chemotherapy in early-responding breast cancer: phase III randomized GeparTrio study. J Natl Cancer Inst. 2008;100:552-62.

15. Von Minckwitz G, Kümmel S, Vogel P, Hanusch C, Eidtmann H, Hilfrich J, et al. Neoadjuvant vinorelbine-capecitabine versus docetaxel-doxorubicincyclophosphamide in early nonresponsive breast cancer: phase III randomized gepartrio trial. J Natl Cancer Inst. 2008;100:542-51.

16. Becht E, Giraldo NA, Lacroix L, Buttard B, Elarouci N, Petitprez F, et al. Estimating the population abundance of tissue-infiltrating immune and stromal cell populations using gene expression. Genome Biol. 2016;17:218

17. von Minckwitz G, Blohmer J-U, Raab G, Löhr A, Gerber B, Heinrich G, et al. In vivo chemosensitivity-adapted preoperative chemotherapy in patients with early-stage breast cancer: the GEPARTRIO pilot study. Ann Oncol. 2005;16:56-63.

18. Hatzis C, Pusztai L, Valero V, Booser DJ, Esserman L, Lluch A, et al. A genomic predictor of response and survival following taxane-anthracycline chemotherapy for invasive breast cancer. JAMA. 2011;305:1873-81.

19. Symmans WF, Peintinger F, Hatzis $C$, Rajan R, Kuerer $H$, Valero V, et al. Measurement of residual breast cancer burden to predict survival after neoadjuvant chemotherapy. J Clin Oncol. 2007;25:4414-22.

20. Haibe-Kains B, Desmedt C, Loi S, Culhane AC, Bontempi G, Quackenbush J, et al. A three-gene model to robustly identify breast cancer molecular subtypes. J Natl Cancer Inst. 2012;104:311-25.

21. Yi M, Huo L, Koenig KB, Mittendorf EA, Meric-Bernstam F, Kuerer HM, et al. Which threshold for ER positivity? A retrospective study based on 9639 patients. Ann Oncol. 2014:25:1004-11.

22. Iwamoto T, Booser D, Valero V, Murray JL, Koenig K, Esteva FJ, et al. Estrogen receptor (ER) mRNA and ER-related gene expression in breast cancers that are $1 \%$ to $10 \%$ ER-positive by immunohistochemistry. J Clin Oncol. 2012;30:729-34

23. Kaneko K, Ishigami S, Kijima Y, Funasako Y, Hirata M, Okumura H, et al. Clinical implication of HLA class I expression in breast cancer. BMC Cancer. 2011;11:454.

24. Iwayama Y, Tsuruma T, Mizuguchi T, Furuhata T, Toyota N, Matsumura M, et al. Prognostic value of HLA class I expression in patients with colorectal cancer. World J Surg Oncol. 2015;13:1-7.

25. Kikuchi E, Yamazaki K, Torigoe T, Cho Y, Miyamoto M, Oizumi S, et al. HLA class I antigen expression is associated with a favorable prognosis in early stage non-small cell lung cancer. Cancer Sci. 2007;98:1424-30.

26. Gao Q, Patani N, Dunbier AK, Ghazoui Z, Zvelebil M, Martin LA, et al. Effect of aromatase inhibition on functional gene modules in estrogen receptorpositive breast cancer and their relationship with antiproliferative response. Clin Cancer Res. 2014;20:2485-94.

27. Dunbier AK, Ghazoui Z, Anderson H, Salter J, Nerurkar A, Osin P, et al. Molecular profiling of aromatase inhibitor-treated postmenopausal breast tumors identifies immune-related correlates of resistance. Clin Cancer Res. 2013;19:2775-86.

28. Engels CC, Charehbili A, van de Velde CJH, Bastiaannet E, Sajet A, Putter $H_{\text {, }}$ et al. The prognostic and predictive value of Tregs and tumor immune subtypes in postmenopausal, hormone receptor-positive breast cancer patients treated with adjuvant endocrine therapy: a Dutch TEAM study analysis. Breast Cancer Res Treat. 2015;149:587-96.

\section{Publisher's Note}

Springer Nature remains neutral with regard to jurisdictional claims in published maps and institutional affiliations. 